\section{Multifunctional Composite Nanoparticles: Magnetic, Luminescent, and Mesoporous}

Yu-Shen Lin, ${ }^{\dagger}$ Si-Han Wu ${ }^{\dagger}$ Yann Hung, ${ }^{\dagger}$ Yi-Hsin Chou, ${ }^{\ddagger}$ Chen Chang, ${ }^{\ddagger}$ Meng-Liang Lin,${ }^{\dagger}$ Chih-Pin Tsai, ${ }^{\dagger}$ and Chung-Yuan $\mathrm{Mou}^{*, \#, \$}$

Department of Chemistry and Center for Condensed Matter Sciences, National Taiwan University, Taipei, Taiwan 106, and Institute of Biomedical Sciences, Academia Sinica, Taipei, Taiwan 115

Received August 22, 2006 Revised Manuscript Received September 25, 2006

Multifunctional nanomaterials have recently become an attractive research field. Nanosized materials with unique magnetic and luminescence properties have great potential in biological applications such as MRI contrast agent, drug delivery carrier, cell sorting, and labeling. ${ }^{1}$ Several reports have focused on the synthesis and investigation of bifunctional nanomaterials, such as hybrid ${ }^{2 a-c}$ and core-shell nanocomposites. ${ }^{2 \mathrm{~d}, \mathrm{e}}$

Because mesoporous silicas possess unique properties of high surface area, large pore volume, uniform pore size, ${ }^{3}$ and low cytotoxicity, ${ }^{4 \mathrm{c}}$ multifunctional mesoporous composites that display both magnetic and luminescence functionalities would be very useful in biomedical applications. In our previous works, we reported gadolinium-incorporated nanosized mesoporous silica (Gd-MS) as an MRI contrast agent ${ }^{4 \mathrm{a}}$ and FITC attached mesoporous silica nanoparticles (FITC-MSNs) as cell markers. ${ }^{4 b, c}$ Currently, there are a few reports about the synthesis of magnetic mesoporous silica nanocomposites, but the porous structures are irregular and the particles after calcination unable to disperse in water. ${ }^{5}$ Thus, the application of these magnetic mesoporous silica

\footnotetext{
* Corresponding author. E-mail: cymou@ntu.edu.tw.

$\dagger$ Department of Chemistry, National Taiwan University.

* Institute of Biomedical Sciences, Academia Sinica.

$\S$ Center for Condensed Matter Sciences, National Taiwan University.

(1) (a) Huh, Y.-M.; Jun, Y.-W.; Song, H.-T.; Kim, S.; Choi, J.-S.; Lee, J.-H.; Yoon, S.; Kim, K.-S.; Shin, J.-S.; Suh, J.-S.; Cheon, J. J. Am. Chem. Soc. 2005, 127, 12387. (b) Giri, S.; Trewyn, B. G.; Stellmarker, M. P.; Lin, V. S.-Y. Angew. Chem., Int. Ed. 2005, 44, 5038. (c) Kim, J.; Lee, J. E.; Lee, J.; Jang, Y.; Kim, S.-W.; An, K.; Yu, J. H.; Hyeon, T. Angew. Chem., Int. Ed. 2006, 45, 4789. (d) Sen, T.; Sebastianelli, A.; Bruce, I. J. J. Am. Chem. Soc. 2006, 128, 7130.
}

(2) (a) Yu, H.; Chen, M.; Rice, P. M.; Wang, S. X.; White, R. L.; Sun, S Nano Lett. 2005, 5, 379. (b) Shi, W.; Zeng, H.; Sahoo, Y.; Ohulchanskyy, T. Y.; Ding, Y.; Wang, Z. L.; Swihart, M.; Prasad, P. N. Nano Lett. 2006, 6, 875. (c) Salgueiriño-Maceira, V.; Correa-Duarte, M. A.; Spasova, M.; Liz-Marzán, L. M.; Farle, M. Adv. Funct. Mater. 2006, 16, 509. (d) Yi, D. K.; Selvan, S. T.; Lee, S. S.; Papaefthymiou, G. C.; Kundaliya, D.; Ying, J. Y. J. Am. Chem. Soc. 2005, 127, 4990. (e) Yoon, T.-J.; Yu, K. N.; Kim, E.; Kim, J. S.; Kim, B. G.; Yun, S.-H.; Sohn, B.-H.; Cho, M.-H.; Lee, J.-K.; Park, S. B. Small 2006, 2, 209.

(3) Beck, J. S.; Vartuli, J. C.; Roth, W. J.; Leonowicz, M. E.; Kresge, C. T.; Schmitt, K. D.; Chu, C. T.-W.; Olson, D. H.; Sheppard, E. W.; McCullen, S. B.; Higgins, J. B.; Schlenker, J. L. J. Am. Chem. Soc. 1992, 114, 10834.

(4) (a) Lin, Y.-S.; Hung, Y.; Su, J.-K.; Lee, R.; Chang, C.; Lin, M.-L.; Mou, C.-Y. J. Phys. Chem. B 2004, 108, 15608. (b) Lin, Y.-S.; Tsai, C.-P.; Huang, H.-Y.; Kuo, C.-T.; Huang, D.-M.; Chen, Y.-C.; Mou, C.-Y. Chem. Mater. 2005, 17, 4570. (c) Huang, D.-M.; Hung, Y. Ko, B.-S.; Hsu, S.-C.; Chen, W.-H.; Chien, C.-L.; Tsai, C.-P.; Kuo, C.-T.; Kang, J. C.; Yang, C.-S.; Mou, C.-Y.; Chen, Y.-C. FASEB J. 2005, 19, 2014.
Scheme 1. Synthetic Procedure of Tumblerlike Mesoporous Silica Nanocomposites (Mag-Dye@MSN)

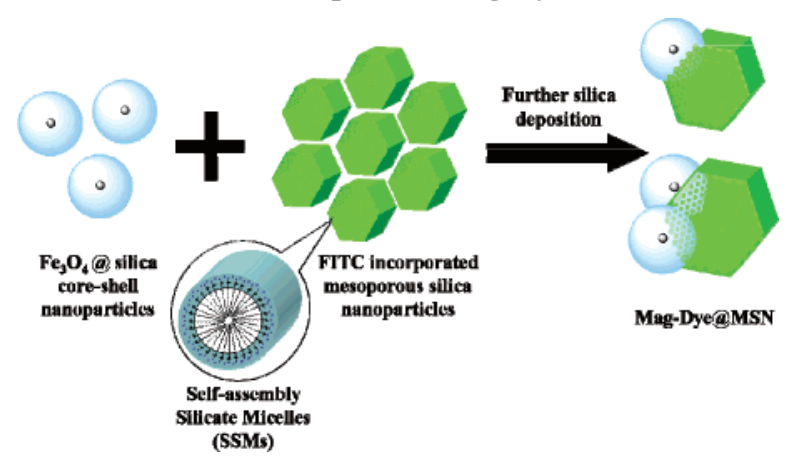

nanocomposites in biological systems is limited. Herein, we develop a new strategy for the synthesis of uniform magnetic/ luminescent functional mesoporous silica nanoparticles with well-ordered porous structure and good aqueous dispersity. Furthermore, we evaluate its potential in biological applications. Preliminary results in the cell uptake experiments are shown.

The synthetic procedure of uniform tumblerlike mesoporous silica nanoparticles (Mag-Dye@MSN) is illustrated in Scheme 1. The first step is to synthesize size-controlled superparamagnetic nanocrystals $\left(\mathrm{Fe}_{3} \mathrm{O}_{4}\right)$. Monodispersed $\mathrm{Fe}_{3} \mathrm{O}_{4}$ nanocrystals (synthesized by Sun's route) are obtained by a high-temperature decomposition of $\mathrm{Fe}(\mathrm{acac})_{3}$ and suspended in hexane (see the Supporting Information, Figure S1). ${ }^{6}$ In the second part, the magnetic nanoparticles are coated with a silica shell by hydrolysis of TEOS in oil-inwater reverse microemulsion ${ }^{7}$ to obtain water-suspended magnetic core-shell nanoparticles $\left(\mathrm{Fe}_{3} \mathrm{O}_{4} @ \mathrm{SiO}_{2}\right.$, see the Supporting Information, Figure S2). Details of the synthetic methods of $\mathrm{Fe}_{3} \mathrm{O}_{4}$ and $\mathrm{Fe}_{3} \mathrm{O}_{4} @ \mathrm{SiO}_{2}$ are described in the Supporting Information. During the synthetic process, organic dyes can be simultaneously incorporated into the mesoporous silica framework by the co-condensation method. ${ }^{4 b}$ Multifunctional mesoporous nanoparticles are prepared by adding organic dyes and $\mathrm{Fe}_{3} \mathrm{O}_{4} @ \mathrm{SiO}_{2}$ to an ammonia solution containing dilute tetraethyl orthosilicate (TEOS) and a low surfactant concentration. Hence, $0.1 \mathrm{~g}$ of cetyltrimethylammonium bromide $\left(\mathrm{C}_{16} \mathrm{TAB}\right)$ was dissolved in $45 \mathrm{~g}$ of 0.51 $\mathrm{M} \mathrm{NH}_{4} \mathrm{OH}$ at $50{ }^{\circ} \mathrm{C}$, and $0.5 \mathrm{~mL}$ of $0.2 \mathrm{M}$ dilute TEOS (in ethanol) was added with vigorous stirring. After the solution was stirred for $1 \mathrm{~h}, 0.4 \mathrm{~mL}$ of preconjugated $N$-1-(3trimethoxy-silylpropyl)- $N$-fluoresceyl thiourea (FITC- APTMS), $5 \mathrm{~mL}$ of $\mathrm{Fe}_{3} \mathrm{O}_{4} @ \mathrm{SiO}_{2}$ colloidal solution, and $0.4 \mathrm{~mL}$ of $0.88 \mathrm{M}$ dilute TEOS were added sequentially under vigorous stirring. The solution gradually turned turbid and was aged under static condition at $50{ }^{\circ} \mathrm{C}$ for $24 \mathrm{~h}$. The as-

(5) (a) Zhao, W.; Gu, J.; Zhang, L.; Chen, H.; Shi, J. J. Am. Chem. Soc 2005, 127, 8916. (b) Kim, J.; Lee, J. E.; Lee, J.; Yu, J. H.; Kim, B. C.; An, K.; Hwang, Y.; Shin, C.-H.; Park, J.-G.; Kim, J.; Hyeon, T. J. Am. Chem. Soc. 2006, 128, 688. (c) Yi, D. K.; Lee, S. S.; Papaefthymiou, G. C.; Ying, J. Y. Chem. Mater. 2006, 18, 614.

(6) Sun, S.; Zeng, H. J. Am. Chem. Soc. 2002, 124, 8204.

(7) Santra, S.; Bagwe, R. P.; Dutta, D.; Stanley, J. T.; Walter, G. A.; Tan, W.; Moudgil, B. M.; Mericle, R. A. Adv. Mater. 2005, 17, 2165. 

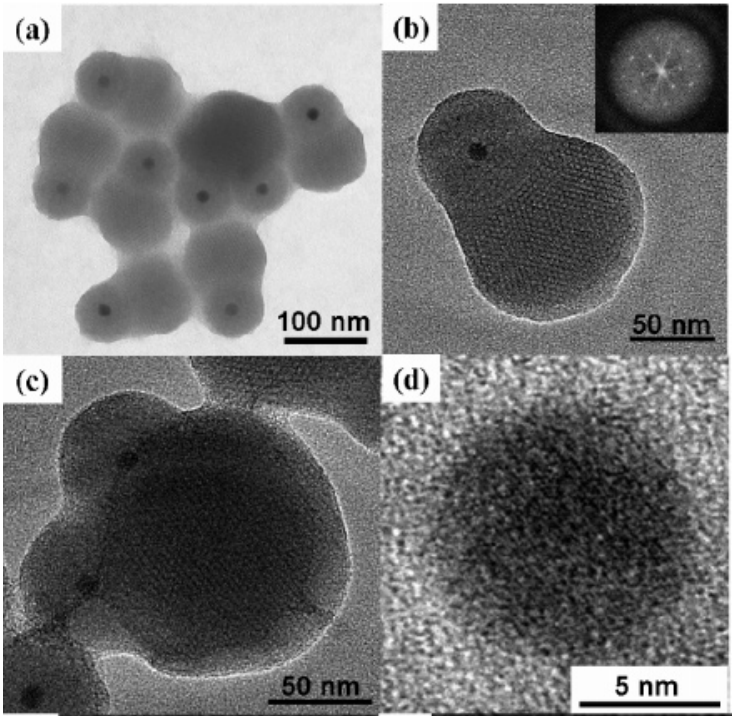

(d)

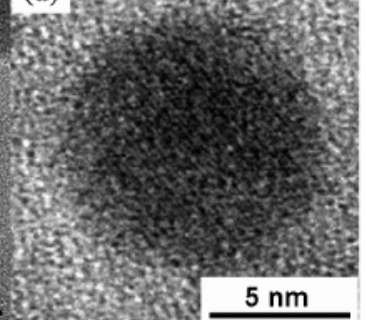

(e)

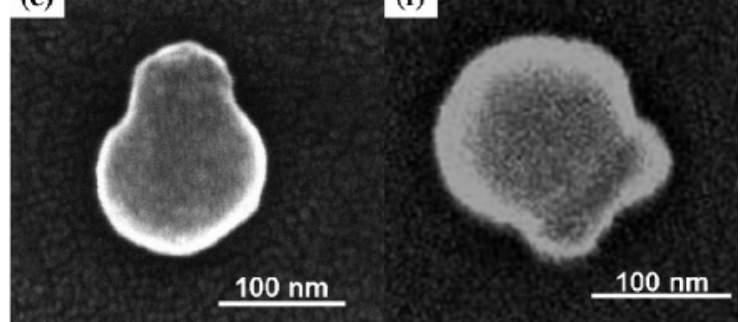

Figure 1. (a) TEM, (b, c) HR-TEM, and (e, f) FE-SEM images of MagDye@MSN. The inset of (b) is the Fourier transform pattern of porous structure. (d) Crystallinity of $\mathrm{Fe}_{3} \mathrm{O}_{4}$ inside extracted Mag-Dye@MSN.

synthesized particles were centrifuged at $12000 \mathrm{rpm}$ for 20 min and then washed and redispersed in ethanol or water. The surfactants were removed by a fast and efficient ionexchange method ${ }^{8}\left(0.3 \mathrm{~g}\right.$ of $\mathrm{NH}_{4} \mathrm{NO}_{3}$ in $150 \mathrm{~mL}$ of ethanol) and examined by FTIR (see the Supporting Information, Figure S3). The nanoparticle solution used for relaxivity measurements and cellular uptake experiments was prepared by transferring the extracted Mag-Dye@MSN from ethanol to D.I. water.

TEM (Figure 1a) and FE-SEM (Figure 1e) images revealed that the multifunctional nanoparticles not only possess a unique tumblerlike morphology but are also very uniform in size. Each Mag-Dye@MSN was formed by fusing one (Figure 1b) or two (Figure 1c) $\mathrm{Fe}_{3} \mathrm{O}_{4} @ \mathrm{SiO}_{2}$ and one mesoporous silica nanoparticle. The average dimensions of Mag-Dye@MSN are $154 \mathrm{~nm}$ in the long axis and $115 \mathrm{~nm}$ in the short axis. Images $\mathrm{c}$ and $\mathrm{f}$ of Figure 1 also show that some particles (9\% of total) are composed of two $\mathrm{Fe}_{3} \mathrm{O}_{4} @ \mathrm{SiO}_{2}$ nanoparticles, resulting in a funny morphology that looks like a frog. The HR-TEM image (Figure 1b) of MagDye@MSN and the Fourier transform pattern simultaneously show that the Mag-Dye@MSN exhibit a well-ordered porous structure with a 2D hexagonal arrangement. The HR-TEM image (Figure 1d) and XRD pattern (see the Supporting Information, Figure S4) of Mag-Dye@MSN both indicate that the magnetic nanocrystal embedded in silica still retains its original crystallinity after silica coating and surfactant extraction.

(8) Lang, N.; Tuel, A. Chem. Mater. 2004, 16, 1961.
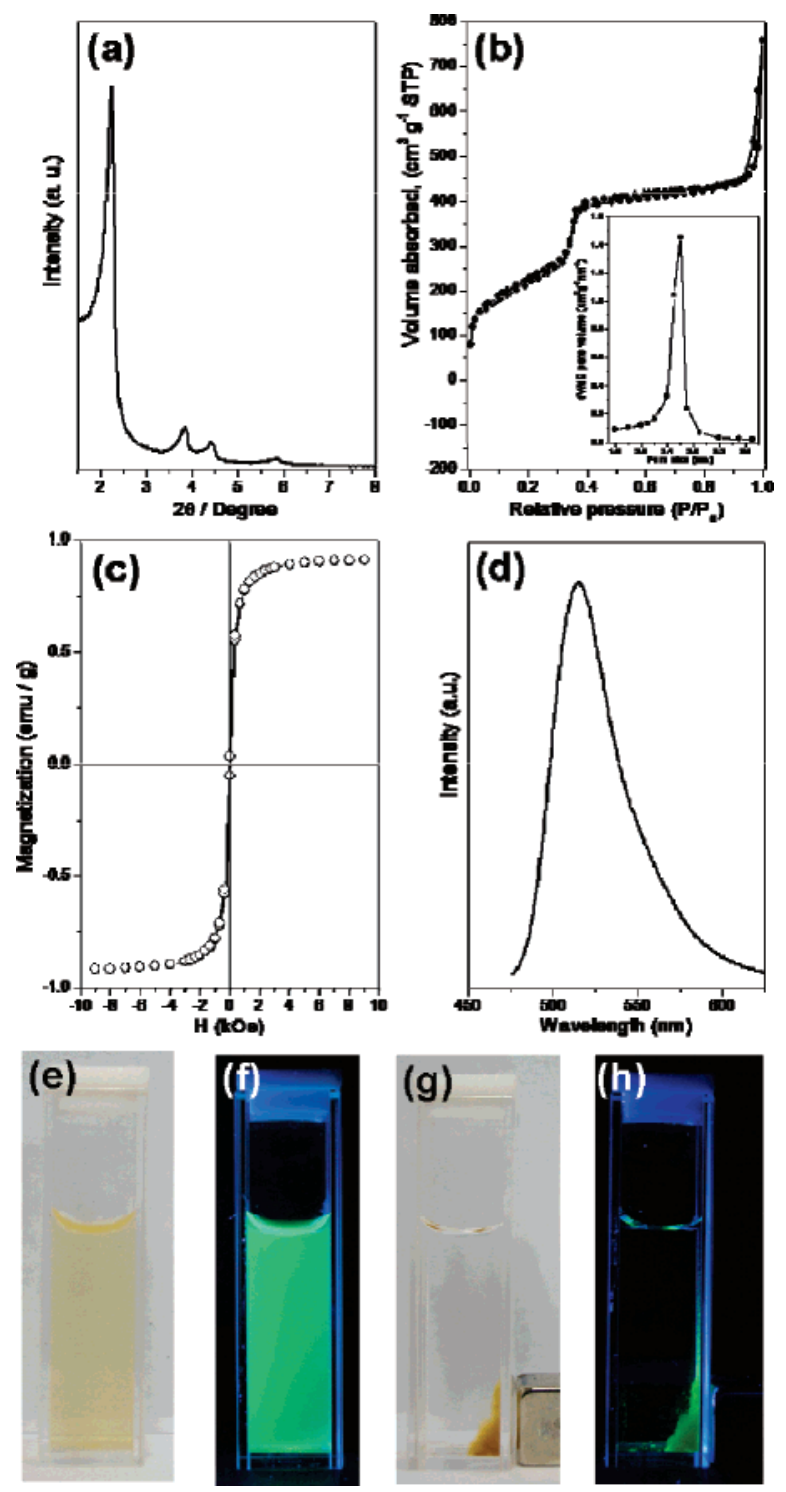

Figure 2. (a) XRD pattern, (b) $\mathrm{N}_{2}$ adsorption-desorption isotherms (inset: $\mathrm{BJH}$ pore size distribution), and (c) field-dependent magnetization curve of extracted Mag-Dye@MSN at $300 \mathrm{~K}$. (d) PL spectrum $\left(\lambda_{\mathrm{ex}}=\right.$ $450 \mathrm{~nm}$ ) of water-suspended Mag-Dye @MSN. Photographs of (e) aqueous suspension of Mag-Dye@MSN, (f) after UV light irradiation, (g) after magnetic capture, and (h) UV light irradiation.

We reasoned the formation mechanism of Mag-Dye@MSN as follows. When the dilute TEOS was added to the aqueous ammonia solution containing low surfactant concentrations of $\mathrm{C}_{16} \mathrm{TAB}$, self-assembled silicate micelles (SSMs) formed. ${ }^{9}$ As TEOS and FITC-APTMS further hydrolyzed, silica further deposited on the SSMs. Simultaneously, the nearby $\mathrm{Fe}_{3} \mathrm{O}_{4} @ \mathrm{SiO}_{2}$ coalesced with SSMs, forming the tumblerlike structured nanoparticles. These nanoparticles not only combine magnetic and luminescence properties but also possess well-ordered porosity.

The XRD pattern (Figure 2a) shows a well-ordered 2D hexagonal mesoporous structure. The $\mathrm{N}_{2}$ adsorption-desorption isotherms (Figure 2b) exhibit a characteristic type IV isotherm, and no hysteresis loop is observed. The pore size $(2.5 \mathrm{~nm})$ of extracted Mag-Dye @MSN is obtained by

(9) Cai, Q.; Luo, Z.-S.; Pang, W.-Q.; Fan, Y.-W.; Chen, X.-H.; Cui, F.Z. Chem. Mater. 2001, 13, 258. 
BJH calculation, as shown in the inset of Figure $2 b$. The surface area and pore size of Mag-Dye@MSN are $828 \mathrm{~m}^{2}$ $\mathrm{g}^{-1}$ and $2.5 \mathrm{~nm}$, respectively. The SQUID spectrum (Figure 2c) measured at $300 \mathrm{~K}$ exhibits no hysteresis loop, which means that Mag-Dye@MSN still possess the superparamagnetic property after surfactant extraction. PL spectrum (Figure 2d) shows the typical fluorescence emission of FITC. It is easy to change the color of luminescence by incorporating different dyes into silica frameworks. Digital photographs of an aqueous suspension of Mag-Dye@MSN before and after UV light irradiation (images e and f, respectively, of Figure 2) show that Mag-Dye@MSN is well-dispersed in water. Dynamic light scattering (DLS) measurements give an average size of $150 \mathrm{~nm}$, confirming that Mag-Dye@MSN is well-dispersed in water (see the Supporting Information, Figure S5). Besides, the magnetic separation of MagDye@MSN was tested by applying a magnet near the cuvette (Figure 2g). It is obvious that the green fluorescence of FITC is detected only next to the magnet (Figure 2h). This observation further proves that the core-shell $\mathrm{Fe}_{3} \mathrm{O}_{4} @ \mathrm{SiO}_{2}$ and FITC are combined together.

Because Mag-Dye@MSN is well-dispersed in water, $T_{2^{-}}$ weighted MR images (MRI) are taken at $4.7 \mathrm{~T}$ on a Biospec spectrometer without dispersion aids. It is overt that the image (Figure 3a) darkens as the amount of Mag-Dye@MSN increases. The $T_{2}$-enhancing capability $\left(r_{2}, T_{2}\right.$ relaxivity) of Mag-Dye@MSN is also measured at 0.47 T on a Minispec spectrometer. The $T_{2}$ relaxivity (the slope obtained by plotting $1 / T_{2}$ versus $\mathrm{Fe}^{3+}$ content, Figure $3 \mathrm{~b}$ ) of Mag-Dye@MSN is $r_{2}=153 \mathrm{mM}^{-1} \mathrm{~S}^{-1}$ (The Fe ${ }^{3+}$ content of Mag-Dye@MSN is determined by ICP-AES). This is a rather good contrast agent for $T_{2}$-weighted MR images. To explore the biological applications of Mag-Dye@MSN, cellular uptake of extracted Mag-Dye@MSN was investigated by confocal microscope. Merged confocal images of NIH 3T3 fibroblast cells (Figure 3c) show that Mag-Dye@MSN have been internalized into cells. This implicates that Mag-Dye@MSN is a potential biomarker for multimodal imaging. Details of MRI and cellular uptake experiments are described in the Supporting Information. The cytoxicity of Mag-Dye@MSN on NIH 3T3 cells was examined by a Trypan Blue exclusion assay (see the Supporting Information, Figure S6). The results indicate that the cell viability was not affected up to $200 \mu \mathrm{g} / \mathrm{mL}$ of Mag-Dye@MSN.

In conclusion, we have developed a strategy for the synthesis of a multifunctional nanomaterial. Mag-Dye@MSN simultaneously possesses magnetic, luminescence, and porous properties. We have further measured the relaxivity and taken phantom images of Mag-Dye@MSN in the MRI $T_{2}$
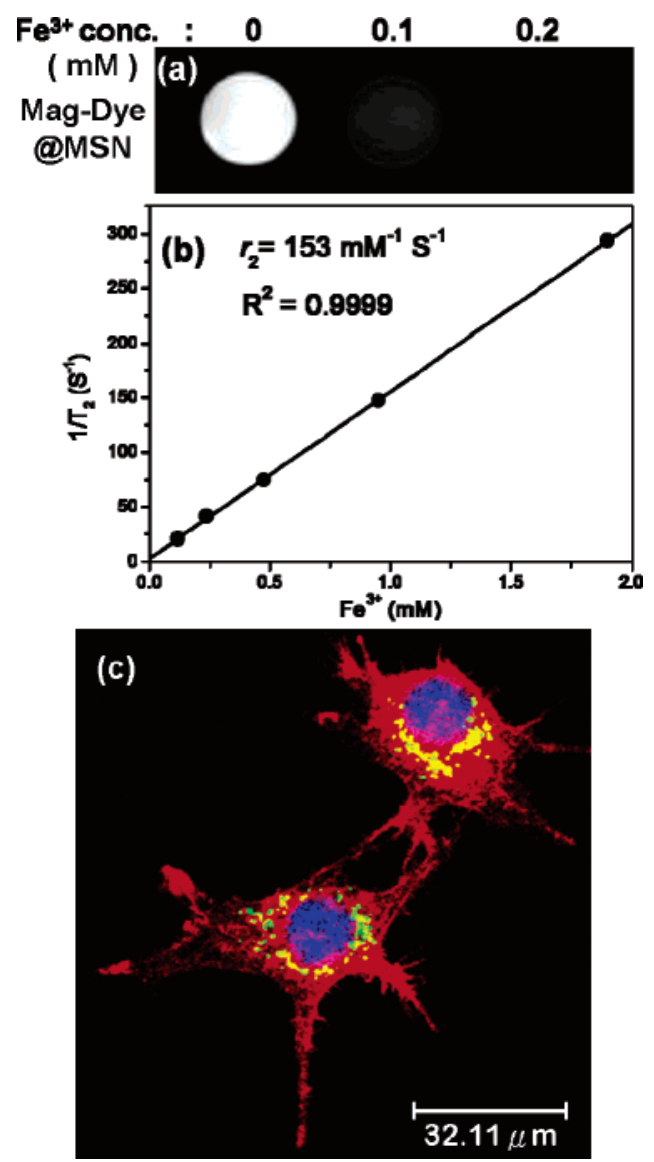

Figure 3. (a) $T_{2}$-weighted MR images of an aqueous suspension of MagDye@MSN taken at $4.7 \mathrm{~T}$ on a Biospec spectrometer; (b) $T_{2}$ relaxivity plot of an aqueous suspension of Mag-Dye@MSN measured at $0.47 \mathrm{~T}$ on a Minispec spectrometer. (c) Merged confocal images of Mag-Dye@MSN in NIH 3 T3 cells after $1 \mathrm{~h}$ of uptake time; cell skeleton was stained with rhodamine phalloidin (red), and cell nucleus with DAPI (blue).

mode and demonstrated that Mag-Dye@MSN is able to internalize into NIH 3 T3 cells without any other uptakeenhancing techniques. These porous nanoparticles have great potential in cell tracking and drug delivery.

Acknowledgment. This research was supported by a grant from the National Science Council of Taiwan. Technical support from the Functional and Micro-Magnetic Resonance Imaging Center supported by the National Research Program for Genomic Medicine, Taiwan, is acknowledged. We thank Dr. YaoHung Tseng for his help with FE-SEM measurements.

Supporting Information Available: Detailed synthesis, TEM images, FTIR, XRD spectra, dynamic light scattering, and cell viability in PDF format. This material is available free of charge via the Internet at http://pubs.acs.org.

CM061976Z 